\title{
Permeability and reactivity of cemented paste backfill with sodium silicate
}

\author{
Hoda Mohammad Pour, Mamadou Fall \\ Department of Civil Engineering, University of Ottawa \\ 161 Louis Pasteur, K1N 6N5, Ottawa, ON, Canada \\ hmoha043@uottawa.ca
}

\begin{abstract}
The key properties of sodium silicate-bearing cemented paste backfill (S-CPB) that have environmental impacts are investigated in this study. These properties include the permeability and reactivity of S-CPB. S-CPB consists of tailings, water and binder, along with the addition of sodium silicate, which is an alkali activator. The S-CPB samples are prepared and cured for 90 days. The reactivity of the S-CPB samples with sulphidic-tailings ( $45 \%$ wt. pyrite) is investigated by performing oxygen consumption (OC) tests, whereas the permeability of the S-CPB is evaluated through saturated hydraulic conductivity tests. The results of the OC tests show that the addition of $0.3 \%$ wt. of SS to CPB can reduce its reactivity due to an increase in the formation of cement hydration products in the $\mathrm{CPB}$. Furthermore, the results show that SS reduces the hydraulic conductivity of the S-CPB by enhancing or refining its microstructure. The results obtained suggest that the addition of SS to CPB would reduce its environmental impacts.
\end{abstract}

Keywords: Cemented paste backfill, Tailings, Mine, Acid mine drainage, Reactivity, Permeability.

\section{Introduction}

Among the different options for mine waste management, cemented paste backfilling has become one of the most important methods in mining operations around the world due to its technical, economic and environmental benefits. Cemented paste backfill (CPB) is a cementitious material produced by mixing tailings with a solid percentage between $70 \%$ and $85 \%$, water that is either fresh or mine processed, and a hydraulic binder, which is usually $3 \%$ to $7 \%$ by weight. These components are typically combined and mixed on the surface of the mine and transported (by gravity and/or pumping) to fill underground mine voids (stopes), provide underground mine support, and maximize ore recovery. CPB is also considered to be a method of disposing mine waste (tailings). The performance of CPB primarily depends on its mechanical stability cost (depends on the binder consumption), and impacts on the environment, which are commonly determined by its permeability, reactivity and leachability.

The most common type of binder used in the preparation of CPB is Portland cement (PC). PC is not only a costly binder that can represent up to $80 \%$ of the cost of the $\mathrm{CPB}$, but its production is highly energy-intensive and thus generates a large amount of carbon dioxide $\left(\mathrm{CO}_{2}\right)$ in the process. In sum, these factors have compelled mining companies to seek for alternatives that would increase the strength of the $\mathrm{CPB}$, but reduce the cement content and carbon footprint of the mining industry. Sodium silicate is one of the most recent chemical additives that is used to reduce the binder content in $\mathrm{CPB}$ and increase its strength. This new product is dubbed sodium silicate cemented paste backfill (S-CPB), which is a mixture of tailings, water, cement and sodium silicate (SS; usually $0.3-0.4 \%$ by weight of solids). However, the environmental impacts of CPB that contains SS are not well understood. There is therefore a need to address this knowledge gap.

A research program has been conducted at the University of Ottawa for a number of years to assess and understand the environmental properties of CPB that contains SS, such as its permeability (hydraulic conductivity) and reactivity. In this paper, the results of the permeability and reactivity testing of CPB with SS are presented and discussed.

\section{Experimental Program}

\subsection{Materials}

In this study, two types of tailings are used: silica tailings (ST) which are synthetic tailings, and natural tailings (NT) that have been obtained from a hard rock mine in Canada. Portland cement type I (PCI) and blast furnace slag (Slag) were used as the binders. Tap water was used as the mixing water. A commercial pyrite powder with a pyrite content of $45 \%$ wt. 
was mixed with the ST and NT to produce sulphide bearing tailings as pyrite is a sulphide mineral. SS Type N in a liquid form was added to the CPB as an alkaline activator.

\subsection{Sample preparation and mix proportion}

The ingredients of the CPB were mixed in a food mixer for 10 minutes to obtain around 100 homogenous samples. Then, the CPB was poured into cylindrical and polyvinyl moulds of $10 \mathrm{~cm}$ in height and $5 \mathrm{~cm}$ in diameter. They were cured for 90 days. The binder content was fixed at $4.5 \mathrm{wt} \%$ as the percentage of total solids. The activator (SS) content was set at $0.3 \mathrm{wt} \%$, which is based on earlier findings (e.g., Abdul-Hussain 2011) that show that the desired strength of CPB can be achieved with this amount of SS.

\subsection{Testing of samples}

\subsubsection{Oxygen consumption tests}

The tailings in CPB can contain a large amount of sulphide minerals (e.g., pyrite, pyrrhotite). When they are in contact with air, these sulphide minerals could be oxidized by oxygen and cause serious environmental problems (e.g., acid mine drainage (AMD)) with financial ramifications. AMD is considered to be one of the most serious economic and environmental problems that the mining industry is facing worldwide. Furthermore, the oxidation of these sulphide minerals can produce sulphate ions in the CPB. These ions can deteriorate the CPB through sulphate attacks. Therefore, the reactivity of sulphide bearing minerals in CPB can be considered as a key indicator of the environmental impacts and durability performance of any CPB system. Hence, the reactivity of the S-CPB samples prepared in this study is assessed by conducting oxygen consumption (OC) tests (Aldhafeeri and Fall, 2016). The reactivity of the CPB samples was measured by using a galvanic cell type oxygen sensor (Model GC33-200, GC Industries) to determine the OC rate (Figure 1). Further details on the OC tests are given in Aldhafeeri and Fall (2016).



Fig. 1: Schematic of OC test chamber with oxygen sensor (Aldhafeeri and Fall, 2016).

\subsubsection{Hydraulic conductivity tests}

Hydraulic conductivity or permeability is one of the key parameters that significantly influences the environmental impacts of any type of CPB (Fall et al., 2009). Indeed, the tendency of CPB to form AMD is not only a function of the type and amount of sulphide minerals that are found in the CPB, but also the ease with which fluids, such as oxygen and water, enter and move through the CPB matrix, i.e. the permeability properties of the CPB (Fall et al. 2009). Moreover, hydraulic conductivity is one of the key factors that governs the rate of groundwater flow through the CPB mass once it is flooded. Namely, hydraulic conductivity significantly influences the leaching potential and transport of contaminants through the CPB to the groundwater (Fall et al. 2009; Levens et al. 1996). Therefore, hydraulic conductivity tests were conducted on the prepared CPB samples with a flexible wall boundary by using a triaxial cell device in accordance with ASTM D5084-00. Each test was repeated at least two times to ensure the repeatability of the results. 


\section{Results and Discussion}

\subsection{Effect of sodium silicate on reactivity of S-CPB}

Typical results of the OC tests on the S-CPB samples are presented in Fig. 2. It can be observed that the CPB samples with SS have lower reactivity values than those without SS. This lower reactivity can be explained by the fact that the SS activates the hydration of the binder, particularly Slag, which leads to the formation of more hydration products. The latter reduce the porosity and refine the pore structure of S-CPB, which in turn decreases the oxygen diffusion and thus the reactivity of CPB (Aldhafeeri and Fall, 2016).



Fig. 2: Effect of sodium silicate (SS) on reactivity of CPB with $(0.3 \% \mathrm{SS})$ and without (0\% SS) SS (ST: CBP made with silica tailings; NT: CBP made with natural tailings).

\subsection{Effect of sodium silicate on permeability of CPB with sodium silicate}

Typical results of the effect of SS on the saturated hydraulic conductivity $\left(\mathrm{k}_{\mathrm{sat}}\right)$ are depicted in Fig 3 . It can be seen that the value of $\mathrm{K}_{\mathrm{sat}}$ in the samples without SS (NT-0 SS and ST-0 SS) is higher than that of the samples with SS (NT-0.3 SS and ST-0.3 SS). This means that SS reduces the permeability of CPB, which contributes to reducing its environmental impacts. The reason for the reduction in permeability is that SS activates binder hydration, which produces more hydration products, and thus refines the pore structure of $\mathrm{CPB}$, as explained previously. 




Fig. 3: Effect of sodium silicate (SS) on saturated hydraulic conductivity of CPB.

\section{Conclusions}

The influence of SS on two key properties of CPB that have environmental impacts is presented in this paper. The results obtained show that the addition of SS to CPB reduces the reactivity and permeability of CPB. In other words, the addition of SS would reduce the environmental impacts of CPB and therefore benefits the environment. Further tests are being currently conducted at the University of Ottawa to provide a more comprehensive understanding and quantification of the effect of SS on the environmental impacts of CPB.

\section{References}

[1] N. Abdul-Hussain, "Experimental study on the engineering properties of gelfill," Master thesis, University of Ottawa, 2011.

[2] Z. Aldhafeeri, M. Fall, "Temperature dependence of the reactivity of cemented paste backfill," Applied Geochemistry, vol. 72, pp. 10-19, 2016.

[3] M. Fall, D. Adrien, J. C. Celestin, M. Pokharel, M. Touré, "Saturated hydraulic conductivity of cemented paste backfill," Minerals Engineering, vol. 22, no. 15, pp. 1307-1317, 2009.

[4] R. L. Levens, A. D. Marcy, C. M. K. Boldt, "Environmental impacts of cemented mine waste backfill," RI 9599, United States Bureau of Mines, pp. 23, 1996. 